\title{
Now Trending: Using Google Trends to Complement Traditional Epidemiological Methods Used for IBS Research
}

\author{
David J. Levinthal ${ }^{1}$
}

Published online: 18 June 2020

(c) Springer Science+Business Media, LLC, part of Springer Nature 2020

Irritable bowel syndrome (IBS) is a common functional gastrointestinal (GI) disorder characterized by the core symptoms of chronic abdominal pain and disordered bowel movements that are present in the absence of obvious structural or inflammatory disease [1]. The diagnostic criteria for IBS have changed over the years, culminating in the widely accepted Rome Criteria, now in its fourth version [2]. The exact prevalence of IBS in adults remains somewhat elusive, as the changing criteria for IBS have directly impacted traditional epidemiological methods (i.e. large, cross-sectional surveys) that seek to determine this information [3-5]. It seems likely that the recent shift to the more stringent Rome IV criteria definition of IBS may yield a lower population prevalence estimate for IBS than was determined using previous criteria [5]. No matter how one defines IBS, however, studying large numbers of patients using traditional epidemiological methods is a resource-intensive approach. As a consequence, there remain substantial gaps in the understanding of regional differences in the prevalence of IBS around the world. Those living in African nations and smaller countries have been particularly unrepresented in past surveys [3].

Yet, one thing is certain - the human suffering that comes from living with IBS-like illnesses is substantial. Patients with IBS symptoms are moved to address them in a myriad of ways, reflecting their personal preferences, surrounding social structures and culture, and access to health care. In particular, the current era of increasing worldwide internet access and the rapidly growing online presence of health information has fundamentally shaped the IBS patient experience. There simply is much more information available online regarding IBS diagnosis and treatment options now

David J. Levinthal

levinthald@upmc.edu

1 Department of Medicine, Division of Gastroenterology, Hepatology, and Nutrition, University of Pittsburgh School of Medicine, M2 C-wing PUH, 200 Lothrop Street, Pittsburgh, PA 15213, USA than there was 20 years ago. Not surprisingly, the large "IBS in America" survey, conducted in the fall of 2015, documented that more than half of patients with IBS symptoms used internet resources, such as Google searches or WebMD to help self-manage their symptoms [6]. Still, there likely are substantial cultural variations in how IBS sufferers interact with their healthcare system, their social networks, and even the internet to seek help. The driving concerns of IBS sufferers, particularly among those who don't make frequent contact with the healthcare system or those less likely to be included in traditional epidemiological surveys, remains a major blind spot in IBS research.

To begin filling these gaps in knowledge, Flanagan et al. [7] in this issue of Digestive Diseases and Sciences describe their use of Google Trends, a publicly available, open-access analytic tool provided by Google, to explore worldwide trends in Google-based searches for IBS and related IBS therapies. The metadata provided by Google Trends is structured to facilitate time-based series and country-based analyses. Although the precise methodology is somewhat opaque, Google Trends reports a "relative search volume" (RSV) estimate (score range 0-100) that reflects changes in the frequency of a particular search term over time. This approach also normalizes search volumes according to a nation's population and fluctuations in total internet accessibility and use. One of the attractive benefits of this methodological approach is that the data represent a massive democratization of epidemiological investigation - essentially creating a sample pool that includes all individuals accessing specific Google searches across the world. Yet, the RSV metric does not reflect absolute values in search volumes. Indeed, Flanagan et al. report that there is poor correlation of RSV values with prior estimates of IBS prevalence [7]. This is an important finding that certainly gives one pause in recognizing the limitations of using Google Trends for all epidemiological research. It is clear that this method is not suited to generating proxy estimates of the true prevalence of other GI (or non-GI) medical conditions. 
Fig. 1 Time trends in the worldwide relative search volume (RSV) for the keywords "Google Trends" between May 26, 2019, and May 22, 2020.

Solid blue line-Variation in RSV over time. Dotted blue line-linear 'trendline' of RSV values

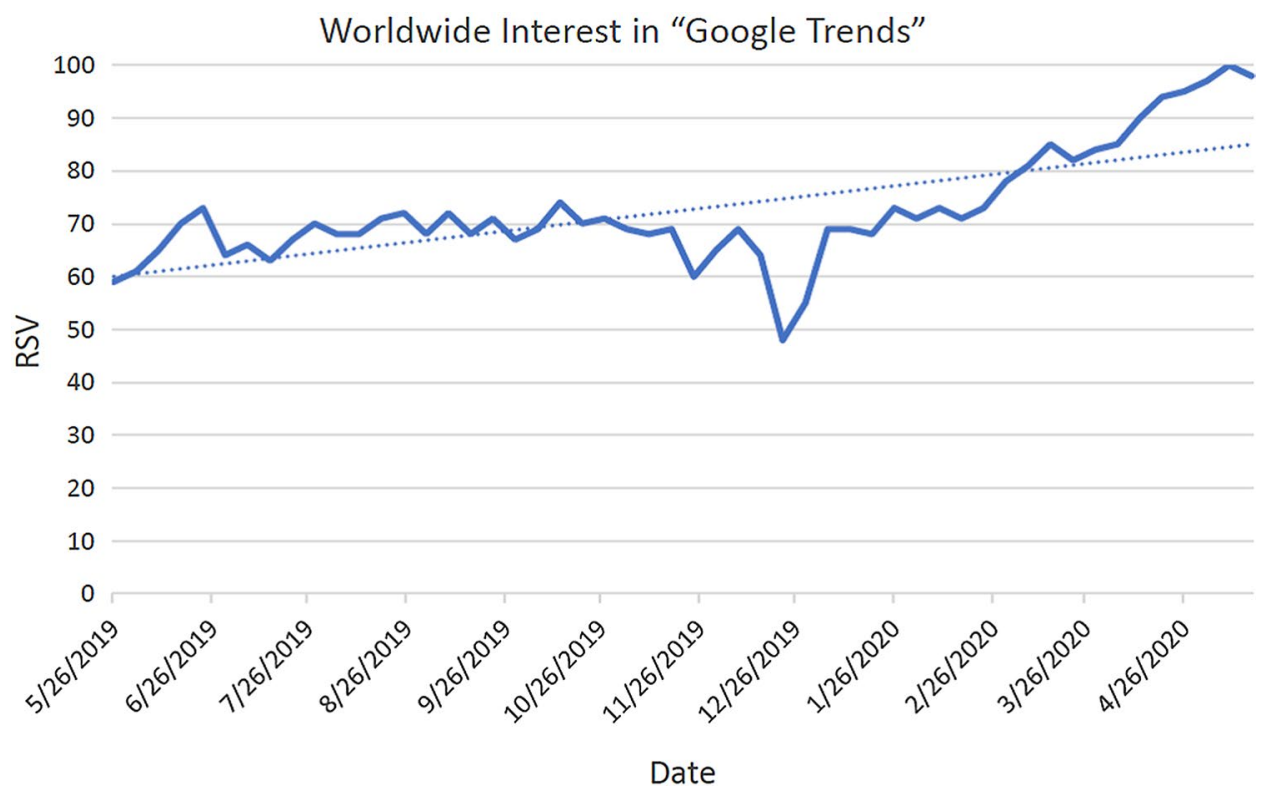

Nevertheless, the Google Trends methodology does clearly complement traditional, cross-sectional epidemiological studies of IBS. The time trends and cross-nation comparisons reported by Flanagan et al. provide interesting insights into worldwide variations of interest in specific IBS treatments. Of note, there appears to be substantial interest for non-pharmacological approaches to IBS treatment, including dietary interventions (low FODMAP and gluten-free diets) and psychological interventions (cognitive behavioral therapy [CBT] and hypnotherapy). Flanagan et al. report that interest in psychological interventions appears to be fairly stable between 2014 and 2018, with interest in low FODMAP diets increasing substantially over this time (up $117 \%$ ) [7]. Perhaps the latter finding is not surprising, given that use of the low FODMAP diet for IBS occurred only over the last several years. Yet the interest in psychological therapies is striking, highlighting a significant disparity in care for IBS patients. It is widely recognized that proportionally few IBS sufferers have ready access to GI psychologists for CBT or hypnotherapy. The results here suggest that patient interest in psychological therapies for IBS appears high, and thus there may not as much patient resistance to this mode of therapy as previously believed. There appears to be significant "pent up demand" for psychological interventions in IBS that will remain challenging to meet. Although the world needs more mental health professionals to directly work with patients, novel digital tools for self-directed CBT [8] have recently shown efficacy in IBS treatment. These digital tools could be efficiently scaled and adapted to different languages and cultures, enabling a more global reach for mental health approaches to IBS treatment that can meet the demand.
The use of Google Trends in academic research will likely accelerate. In fact, interest in Google Trends itself appears to be increasing worldwide over the past year (Fig. 1). On one level, this tool is easy to use, with computations and analysis occurring in seconds that mine complex metadata sets on a scale that would have been nearly inconceivable 20 years ago. Although one must take some caution in interpreting the results of this new form of 'digital epidemiology' [9], internet-based research will undoubtedly take its place astride more traditional epidemiological methods in studying human health in the years to come.

Funding None.

\section{References}

1. Longstreth GF, Thompson WG, Chey WD, Houghton LA, Mearin F, Spiller RC. Functional bowel disorders. Gastroenterology. 2006;130:1480-1491.

2. Lacy BE, Mearin F, Chang L, et al. Bowel disorders. Gastroenterology. 2016;150:1393-1407.

3. Endo Y, Shoji T, Fukudo S. Epidemiology of irritable bowel syndrome. Ann Gastroenterol. 2015;28:158-159.

4. Lovell RM, Ford AC. Global prevalence of and risk factors for irritable bowel syndrome: a meta-analysis. Clin Gastroenterol Hepatol. 2012;10:712-721.

5. Olafur S, Palsson E, Whitehead W, Törnblom H, Sperber AD, Simren M. Prevalence of Rome IV functional bowel disorders among adults in the United States, Canada, and the United Kingdom. Gastroenterology. 2020;158:1262-1273.

6. IBS in America Survey. 2015. http://www.multivu.com/players/ English/7634451-aga-ibs-in-america-survey/docs/survey-findi ngs-pdf-635473172.pdf. Accessed May 22, 2020. 
7. Flanagan R, Kuo B, Staller K. Utilizing Google Trends to assess worldwide interest in irritable bowel syndrome and commonly associated treatments. Dig Dis Sci. (Epub ahead of print). https:// doi.org/10.1007/s10620-020-06290-7.

8. Everitt HA, Landau S, O'Reilly G, on behalf of ACTIB trial group, et al. Assessing telephone-delivered cognitive-behavioural therapy (CBT) and web-delivered CBT versus treatment as usual in irritable bowel syndrome (ACTIB): a multicentre randomised trial. Gut. 2019;68:1613-1623.
9. Cerbellin G, Comelli I, Lippi G. Is Google Trends a reliable tool for digital epidemiology? Insights from different clinical settings. J Epidemiol Glob Health. 2017;7:185-189.

Publisher's Note Springer Nature remains neutral with regard to jurisdictional claims in published maps and institutional affiliations. 